Mahmoud HASSAN ${ }^{1}$

Ahmad SADEK ${ }^{1}$

M. Helmi ATTIA ${ }^{1,2^{*}}$

\title{
INTELLIGENT CYBER-PHYSICAL MONITORING AND CONTROL OF I4.0 MACHINING SYSTEMS - AN OVERVIEW AND FUTURE PERSPECTIVES
}

\begin{abstract}
Rapid evolution in sensing, data analysis, and industrial internet of things technologies had enabled the manufacturing of advanced smart tooling. This has been fused with effective digital inter-connectivity and integrated process control intelligence to form the industry I4.0 platform. This keynote paper presents the recent advances in smart tooling and intelligent control techniques for machining processes. Self-powered wireless sensing nodes have been utilized for non-intrusive measurement of process-born phenomena near the cutting zone, as well as tool wear and tool failure, to increase confidence in the process and tool condition monitoring accuracy. Cyber-physical adaptive control approaches have been developed to optimize the cycle time and cost while eliminating machined part defects. Novel artificial intelligence AI-based signal processing and modeling approaches were developed to guarantee the generalization and practicality of these systems. The paper concludes with the outlook for future work needed for seamless implementation of these developments in industry.
\end{abstract}

\section{INTRODUCTION}

Recently, manufacturers have realized the benefits of advances in digital technologies along with the development of the Industrial Internet of Things (IIoT), where a multitude of devices connected by communication technologies monitors, analyzes, and delivers valuable new insights. The IIoT has expanded at a rapid rate due to the development of smart sensors and data storage capacities that has led to "Industry 4.0" revolution. Advanced manufacturing techniques are combined with IIoT to drive further intelligent actions back in the physical world, motivating unmanned manufacturing. This can result in industrial competitive advantage by reducing cost, increasing productivity, improving part quality, and preventing damage to machined parts during processing. A recent survey that was conducted in Europe showed that more than $90 \%$ of industrial companies are investing in digital factories

\footnotetext{
${ }^{1}$ Aerospace Manufacturing Technology Centre, National Research Council Canada, Canada

${ }^{2}$ Mechanical Engineering Department, McGill University, Canada

*E-mail: helmi.attia@mcgill.ca / helmi.attia@nrc.ca https://doi.org/10.36897/jme/147157
} 
with the aim of increasing the efficiency by $12 \%$ [1]. The Canadian aerospace industry, as an example, has a long-term plan to drive economic growth through innovation, investing approximately $\$ 1.5$ billion yearly in research and development activities [2]. Approximately, $70 \%$ of this sector activities are manufacturing, providing more than $\$ 25$ billion of direct revenue annually. Manufacturing of large aerostructures requires hours of complex metal removing processes. Evaluation of these processes through inspection of the finished workpiece, at the end of the manufacturing cycle, is insufficient since any shortfall cannot be corrected and the product may be scraped. In addition, 7-20\% of total tool machine downtime is caused by tool failure, and the cost of tools and tool changes accounts for 3-12\% of the total processing cost [3]. Proper and reliable manufacturing of aerostructures requires accurate prediction of the condition of the cutting tool and continuous control of the variations of the cutting process. It is clear that improvement and optimization of metal cutting processes are essential for the continuous growth of this sector, which is characterized by high varietylow volume production. Investigations based on advanced Tool Condition Monitoring (TCM) techniques and Adaptive Control (AC) systems are required to achieve this goal of unmanned machining system. Hence, tremendous efforts are exerted towards developing innovative technologies to improve the performance of these systems and to introduce novel approaches that can provide solutions to the challenges facing manufacturers.

An adaptive control (AC) system is a digital controller that adapts itself to the time varying parameters of cutting process dynamics at each control interval [4]. Different approaches have been implemented in adaptive control systems, including model-based and AI approaches, to maintain or optimize a certain performance index, such as process economics, tool life or machined part quality. Cutting forces can change significantly during the course of cutting operations and are directly related to most of the cutting process aspects. Consequently, the bulk of the available research work, as well as commercial systems, have concentrated on adaptive control with constraint type systems, which use real-time feedback to maintain a fixed spindle force/power level. These systems depend on empirical system learning, which requires extensive experimental training and calibration and does not take into account the considerable process variability, as well as the impact of the modified feed on the produced part quality at different tool wear levels.

Several TCM systems have been developed and reported in literature for discrete manufacturing to monitor various aspects of cutting tool deterioration, such as tool wear, chipping, and breakage. These tool failure mechanisms are affected by the cutting conditions and the tool and workpiece materials. As a result, different consequences, such as high cutting forces and temperatures, are expected to affect the deviation in the dimensional accuracy and surface integrity of the machined part. The complexity of the tool deterioration phenomena alongside the high dynamics of cutting processes have limited the opportunities of developing a unique solution to analytically model the complete process [5, 6]. Additionally, the harsh environment of the cutting process has restricted the applicability of real-time direct monitoring, such as using a machine vision system to capture actual geometric changes due to tool wear. An effective real-time sensor-based TCM, therefore, can utilize indirect measurements to put a cutting tool under continuous surveillance to safeguard the workpiece from damage. Sensor selection, multi-sensor fusion, signal processing, feature extraction and selection, and prediction models are the major research topics in indirect TCM systems. 
Different indirect measurements have been utilized and reported in the open literature and correlated to the cutting tool state in machining applications. Cutting forces are considered as the best variable to describe the cutting process due to the completeness of the process information and the sensitivity of the cutting force to tool geometry changes $[3,7,8]$. However, such a measurement is difficult to make during cutting in real industrial environment. On the other hand, the power and current signals of the spindle driving motor can easily be monitored and used to give an indication of the tool condition as they are directly related to the cutting forces $[9,10]$. Acoustic emission AE sensors can capture abnormalities during the turning or milling operations, and have the flexibility to be mounted on the tool or the workpiece without major disturbances to various machining activities $[11,12]$. Worn and broken tools can cause high vibrations during cutting operations; these vibrations can be detected using accelerometers [13-15] Additionally, temperature and ultrasonic sensors have been used to monitor the tool condition and surface roughness, respectively [16-18]. Nevertheless, they did not show their readiness to be applied in industrial applications. The literature on TCM systems has witnessed several contradictions in selecting the appropriate sensor to detect the type(s) of tool failure. This is due to the dependency of the sensor signals on the failure mechanism, the mounting location, orientation, and the tool path, among other factors.

The recent revolution in sensing technologies and wireless communication, as part of the IIOT, has enabled the development of tool-embedded sensors. This allows real-time measurement of primary machining phenomena as close as possible to the signal source. Hence, the acquired signal coherence and accuracy are increased. Miniature wireless accelerometers, strain gauges, polyvinylidene fluoride (PVDF) sensors, and thermocouples have been embedded on either the cutting tool body or the machine spindle to measure the process vibration, torque, deflection, and temperature in milling and other machining operations [19-22]. Such systems have enabled acquiring in-process data near a rotating tool tip. However, several challenges are still to be overcome for industrial implementations of these systems. These include the limited accuracy, sensitivity and bandwidth of miniature sensors and the power management needed to continuously operate over a full working shift. Additionally, most of the available tool-embedded sensors affect the cutting tool rigidity, which may limit their use.

The signal processing methods used in TCM cover the majority of conventional processing techniques, including time domain analysis [3, 23-25], frequency domain analysis [12, 26-29] time-frequency analysis techniques [10, 30-32] and artificial intelligence techniques [14, 30-36]. Despite the tremendous effort exerted in this field, there is still no clear generalized methodology for developing a system to accurately detect the tool condition in real-time, and even worse, to take a proper action. Although several physical and statistical features have been reported as sensitive features to the tool condition, they cannot be generalized as they were extracted from process-dependent data. The level of sensitivity was much dependent on the cutting parameters, acquired signal quality, and signal conditioning and processing method. A smart system should be capable of providing faithful feedback on the state of the monitored process. Additionally, an intelligent system can further take corrective actions based on this feedback to optimize or stop the process, leading to unmanned manufacturing systems. This is achievable through developing hybrid cyber-physical 
monitoring and control systems that utilize analytical physical-based models with AI approaches to deal with the prediction uncertainty in real-time. Such approach is favourable for real-time optimization and control of machining processes through integrating digital shadows with AI monitoring systems to adaptively take corrective actions in real-time. However, to generalize this approach, AI self-learning capability is required for in-process training of monitoring and control systems.

\section{SEAMLESS IMPLEMENTATION OF TCM IN MACHINING SYSTEMS}

\subsection{GENERALIZED SIGNAL PROCESSING AND DECISION-MAKING APPROACH}

The cyclic load in milling processes generates a steady signal that is developed by the periodic engagement and disengagement between tool and workpiece. The characteristics of this signal, e.g., amplitude and frequency, mainly depend on the cutting parameters such as cutting conditions (i.e., speed, feedrate, axial and radial depth of cut), tool geometry and tool path. During a cutting process, progressive tool wear alters the tool edge geometry and hence change the acquired signal properties, which can be distinguished in the frequency and time domains [37, 38]. However, this change is usually distorted in a nonlinear fashion by variations in the cutting parameters and interfering noises in industrial environment. This limits the capability of the features extracted directly from raw signals to globally represent the tool wear state. Recently, Hassan et al. [13] developed a novel signal processing and decision making approach that can mask the effect of cutting parameters and accentuate the tool wear effect in the extracted features. Additionally, this approach greatly minimizes the learning effort needed to train the decision making model and reduces the signal noises effect on the extracted features, which enables its implementation in industrial environment. This generalized approach is based on a two-stage hybrid signal processing technique that integrates time-frequency signal analysis with deep and conventional machine learning approaches. In the first stage, a signal scattering representation technique that fuses wavelet analysis within the convolution neural network layers is adopted. This multi-layer timefrequency analysis technique separates multiscale signal information for low-variant features extraction. It provides locally transposition invariant representation that reduces the extracted features space while preserving the signal energy [39]. Hence, it masks the effect of the cutting conditions variation and the noise on acquired signals, without the loss of valuable tool wear information. To further maximize the variance between tool condition classes over the variance within the extracted features of the same class, a linear discriminant analysis (LDA) structure was developed in the second stage to define the tool condition in real-time. Wavelet-scattering convolution neural network framework (WSCNN) provides stable and invariant signal representation through cascaded layers of tri-step processing framework. Figure 1 illustrates the structure of the developed approach. A moving windowing function is applied to segment the signals. Each segment information is separated among WS framework layers. In the zeroth layer, the segment signal is filtered by low pass filter, which is an averaging scaling function of the signal. The high frequencies information is captured by 
creating a new representation of the same signal segment by applying wavelet transform with each wavelet filter in the filter bank and taking the modulus [39]. These three steps, i.e., scaling, wavelet convolution and modulus, are cascaded in successive layers using the generated segment representation as the input signal. The output of the scaling step of all layers are the scattering features that represent the processed segment. Through these stages, signal deformations, caused by variations in the cutting conditions or the noise, are linearized by separating the variations across different frequency scales [40].

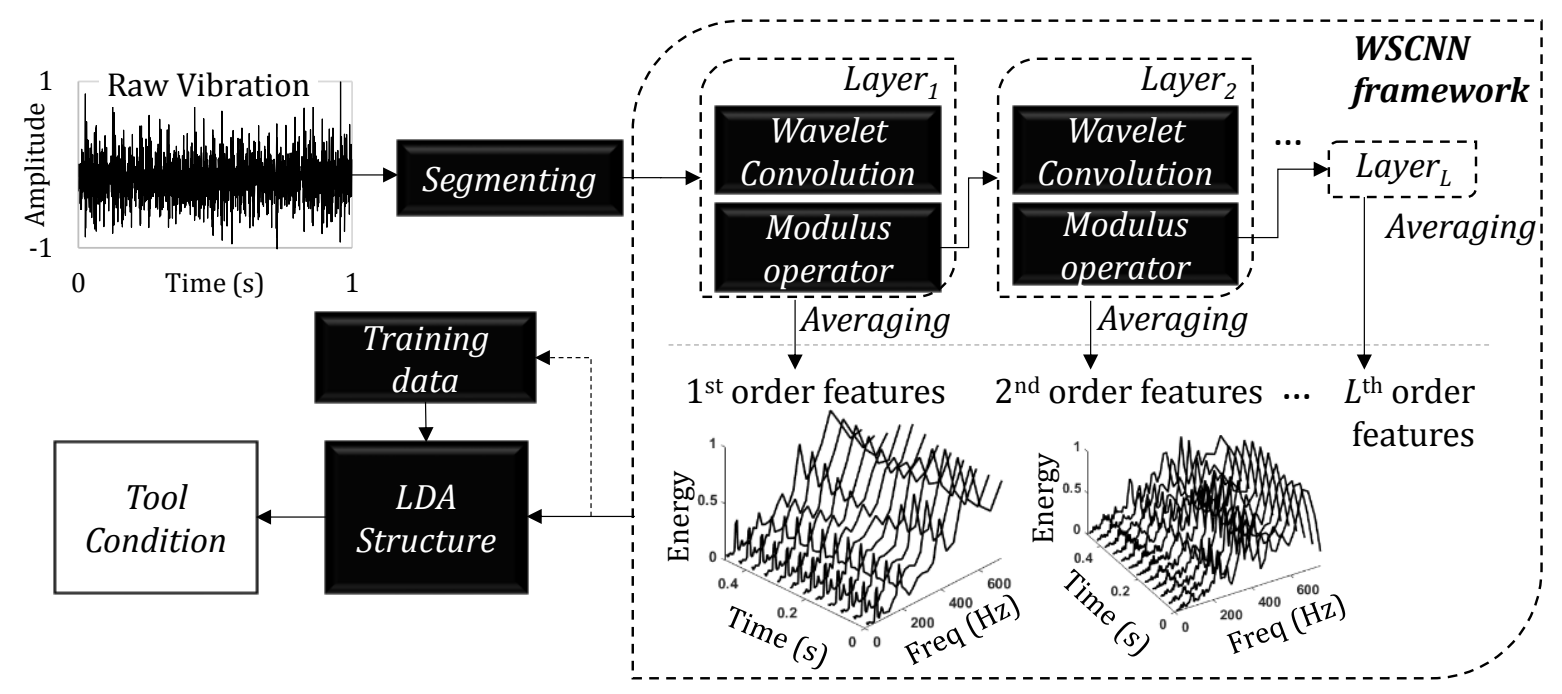

Fig. 1. WSCNN-LDA hybrid approach flow chart [13]

The number of layers, depth of each layer and wavelet function parameters, e.g., type of wavelet and frequency resolution, are predefined parameters that can be adjusted based on the processed signal properties and the required resolution level. This facilitates WSCNN framework for accurate signal representation using low training data, as compared to deep machine learning approaches, where nodes weights and biases need to be learned. Additionally, it eliminates the expert's time and effort for feature extraction and optimization for conventional ML models. A Morlet wavelet, which is a Gaussian windowed sinusoid, is employed in this framework. The wavelet filter banks are automatically selected to have enough frequency resolution to separate harmonic structures based on the passing and rotating frequency of the cutting tool. To generate generalized low-variance features, the scattering features is normalized to de-correlate them at different orders. This approach provides a cutting conditions-independent representation of tool condition, and thus, significantly reduces the required learning effort. An N-way ANOVA analysis of the WSCNN extracted features has shown the features' high sensitivity to the tool condition compared to the cutting conditions (feedrate and depth of cut), tool geometry (number of flutes, diameter, and corner radius) and their interactions [13]. The features provided a distinguished and stable representation of tool wear state over time. The WSCNN-LDA approach has proven to reduce the learning efforts needed to train the classification model to only a couple of seconds of machining using a fresh and a worn tool. This is up to $97 \%$ reduction in learning efforts compared to typical learning approaches. The approach achieved a $98 \%$ detection accuracy 
within more than 500 cutting tests using a wide range of tool paths, where cutting conditions were varied by multiples of up to 10 , in conventional and high speed milling processes of titanium and aluminum alloys.

In [13], the developed approach has been applied to signals acquired from a high-end triaxial accelerometer mounted on the spindle head near the cutting tool, which was wired to a National Instrument data acquisition card type NI 9232. It discussed the system applicability to conditions far from the training data and demonstrated the approach capability to accurately detect the tool condition at different cutting conditions. The results showed that by using only one combination of cutting conditions per tool condition for training, the system accurately detected the tool condition in up to 79 unlearned combinations of cutting conditions for the same tool. Hence, the proposed approach can successfully extrapolate results far from the training dataset.

In addition to changes in cutting parameters, it is known that the frequency response functions of each sensor can have an effect on the vibration signal characteristics. Therefore, in this work, the WSCNN-LDA approach sensitivity to the sensor frequency response is benchmarked through developing and utilizing a cost-effective, self-powered spindlemounted wireless vibration sensor, as shown in Section 2.2.1. Additionally, the practicality and generalization ability of this approach is examined by utilizing different means of sensing and different machine tools. The spindle feedback signals, namely the driving motor current, voltage, and power, acquired from two different machine tools using different transducer types have been analyzed using the WSCNN-LDA approach, as seen in Section 2.2.2. In total, five sensor/machine tool combinations have been tested and benchmarked. The results of applying the WSCNN-LDA approach to these combinations are presented in Section 2.2.3.

\subsection{GENERALIZED SIGNAL PROCESSING AND DECISION-MAKING APPROACH AS A DRIVER FOR DIGITAL TRANSFORMATION}

\subsubsection{IMPLEMENTATION OF AI SENSOR-BASED APPROACHES}

The quality of the acquired vibration signals are highly dependent on the path between the vibration source and the accelerometer location. The use of spindle-mounted accelerometers, which requires invasive embedding for sensor wiring, may limit the machine workspace. Miniature wireless micro-electromechanical system MEMS accelerometers are a cost-effective solution that can be utilized to avoid such machine retrofitting. However, their signals have limited bandwidth and notoriously difficult to filter, making them prone to delivering inaccurate information [41]. The power management of wireless accelerometers adds another layer of complexity as well. The authors' effort to use a self-powered wireless vibration sensing node is presented in this section. Figure 2 shows the developed spindlemounted self-powered vibration-sensing node and its main components. A smart, low power, miniature tri-axial accelerometer (6) with high speed analog-to-digital A/D converter has been used to acquire and digitalize the tool vibration signals. A miniature micro-controller unit (MUC) with built-in wireless transmitter (5) was utilized for signal conditioning and 
transmission. The data is then acquired on a personal computer for processing and TCM. The MCU was powered by a rechargeable battery (3). One of the main drawbacks of available sensing nodes in TCM literature is the need for battery recharging, which interrupt the machining process and require repetitive maintenance actions. To enable self-powering capability, a hybrid energy-harvesting module has been developed using a Radio frequency RF (1) and mechanical energy (2) harvesting components.
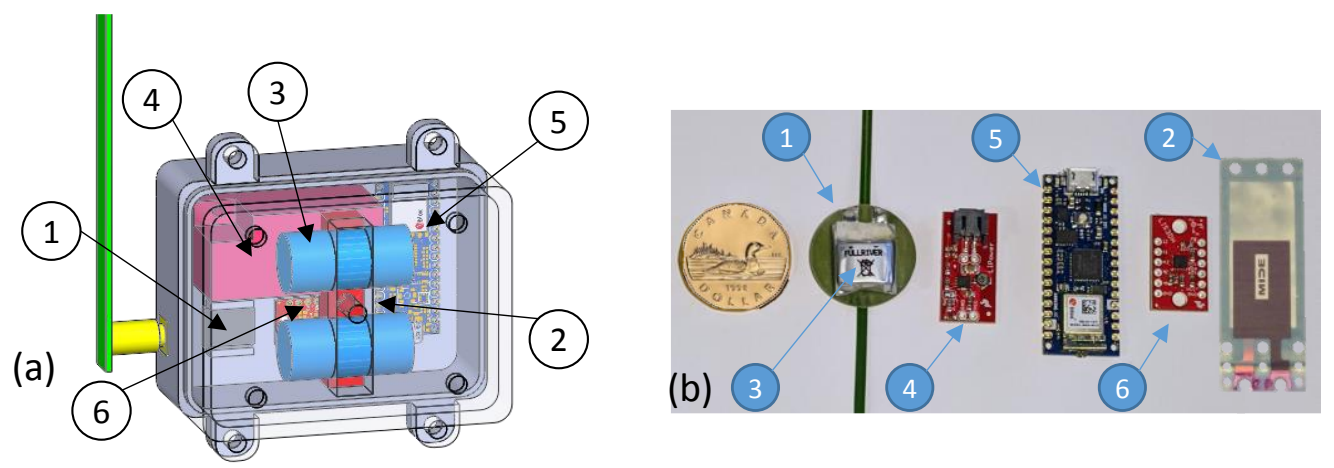

Fig. 2. Wireless spindle-mounted vibration sensing node (a), its components (b)

The RF module harvests the energy from an electromagnetic field generated by an RF transmitter positioned outside the machine tool. The mechanical module converts the machine vibration energy to the electrical energy using piezo ceramics. The harvested energy has been used to charge the powering battery through a power conditioning board (4). The developed node can provide continuous data stream of tri-axial vibration signals for more than eight hours. The MCU can be configured into low power mode during the machine inactive periods to save energy and prolong the battery life. The physical size of the main components of the developed sensing node are shown in Fig. $2 b$ relative to the size of a Lonnie.

\subsubsection{EXPERIMENTAL SETUP AND TEST MATRIX}

In order to assess the performance of the proposed approach, two sets of conventional roughing operations of Ti6Al4V workpiece under flood cooling condition were performed. Table 1 shows the full factorial matrix of the cutting speed $n$, feedrate $f$ and axial $a_{p}$ and radial $a_{e}$ depths of cut. Two tungsten carbide endmills were used; T1 is a four-flute endmill with a $50 \mathrm{~mm}$ diameter and T2 is a three-flute tool with a $32 \mathrm{~mm}$ diameter, which were used in set 1 and set 2 tests, respectively. In total, 192 side-milling tests were performed including one repetition.

Table 1. Cutting conditions full factorial

\begin{tabular}{lllllll}
\hline \hline Set \# & $n(\mathrm{rpm})$ & $f_{z}(\mathrm{~mm} /$ tooth $)$ & $\begin{array}{l}a_{p} \\
(\mathrm{~mm})\end{array}$ & $\begin{array}{l}a_{e} \\
(\mathrm{~mm})\end{array}$ & Direction & Total no. of tests \\
\hline 1 & 300 & $0.1,0.14$ & $3.5,7$ & $12,24,38$ & Up and down milling & 96 \\
2 & 500 & $0.08,0.12$ & $2.5,5$ & $8,16,24$ & Up and down milling & 96 \\
\hline \hline
\end{tabular}


The experimental setup is shown in Fig. 3a. To test the developed approach against different spindle controller and spindle feedback transducers, two machine tools have been utilized. Set 1 tests were performed on a high-power horizontal machining center Makino A88. This machining center is featured with $50 \mathrm{~kW}$ spindle power, three linear and two rotary axes, a maximum spindle speed of $18,000 \mathrm{rpm}$ and a maximum feed rate of $50 \mathrm{~m} / \mathrm{min}$, tool clamping force of $19.6 \mathrm{kN}$, and HSK 100 A spindle adapter. The machine has a FANUC 16i MA controller. The spindle feedback signals, namely the instantaneous current, voltage and power signals were acquired from the spindle-driving module using a BECKHOFF power monitoring card model EL3783. It can measure up to $690 \mathrm{~V}$ voltage and 5 A current with a reaction time, frequency bandwidth and resolution of $50 \mu \mathrm{s}, 5.5 \mathrm{kHz}$ and 16 bits, respectively. Ring-type current dividers, shown in Fig. $3 \mathrm{~b}$ were used to measure and stepdown the primary currents of the spindle driving module while the voltage terminals were connected directly to the EL3783 card for voltage measurement, as shown in the connection scheme in Fig. 3c. A BECKHOFF CX8190 industrial computer with an $800 \mathrm{MHz}$ ARM Cortex ${ }^{\mathrm{TM}}$-A9 processor was used to acquire and store the spindle feedback signals from the EL3783 card through EtherCAT connection.
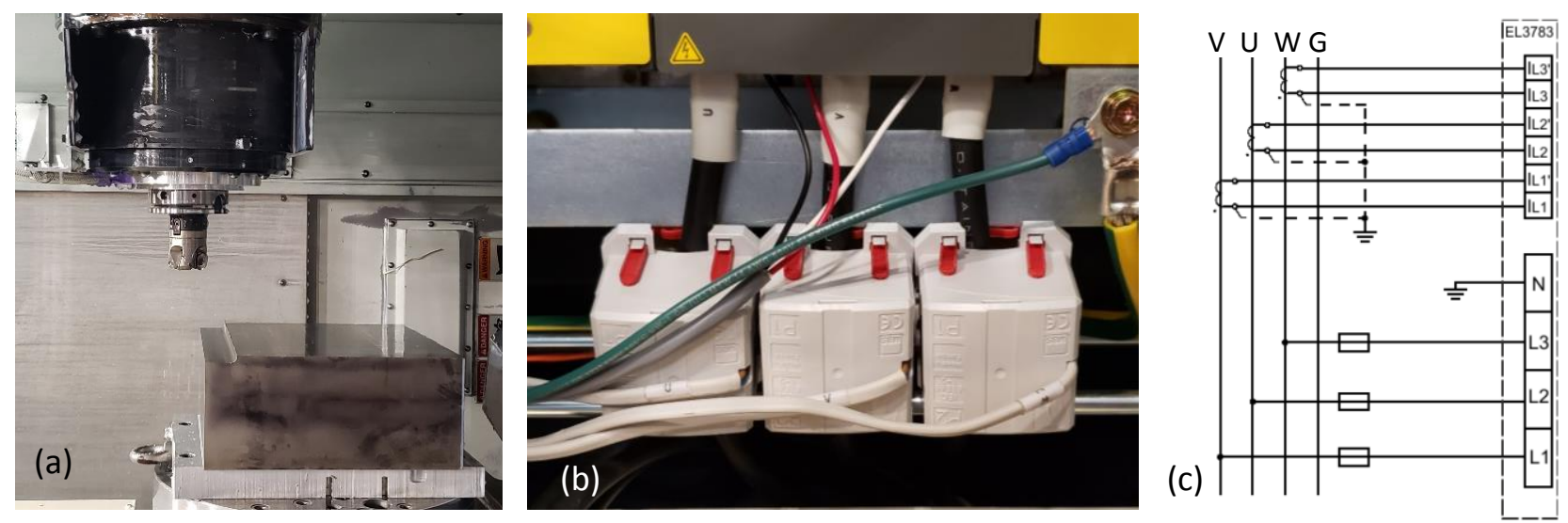

Fig. 3. Experimental setup (a), current dividers (b), DAQ connection scheme (c)

Set 2 tests were performed on a DMU 100P DuoBlock Machining Centre with a maximum spindle speed and power of 18,000 rpm and $28 \mathrm{~kW}$, respectively. LEM Voltage and current transducers type LF 310-S and DVL 1000, respectively, have been used to measure the instantaneous feedback signals of the spindle motor, whereas the instantaneous power was digitally calculated based on the acquired signals. These transducers can measure alternating current, direct current, and pulsed current signals with range, reaction time, and frequency bandwidth of $\pm 500 \mathrm{~A}, 0.5 \mu \mathrm{s}$, and $100 \mathrm{kHz}$, respectively, for the current transducers, and $\pm 1,500 \mathrm{~A}, 30 \mu \mathrm{s}$, and $14 \mathrm{kHz}$, respectively, for the voltage transducers. The transducers were mounted on the three phases between the spindle motor and its pulse-width modulation (PWM) driving module. Simultaneously, tri-axial vibration signals were acquired using a miniature triaxial PCB accelerometer type 356A71, mounted on the spindle head. It has a sensitivity and a frequency range of $1.02 \mathrm{pC} /\left(\mathrm{m} / \mathrm{s}^{2}\right)$ and $4 \mathrm{kHz}$, respectively. A National Instruments data acquisition card type NI 9222 connected to an NI cDAQ 9191 chassis has 
been used to digitalize and store the acquired signals. In addition, the tri-axial vibration signals were acquired using the developed wireless sensing node, which is wirelessly connected to a personal computer for data acquisition.

The flank wear VB was selected as tool life degradation measure. It was divided into three spans: fresh $\mathrm{VB} \leq 0.2 \mathrm{~mm}$, usable $0.2 \mathrm{~mm}<\mathrm{VB} \leq 0.45 \mathrm{~mm}$ and worn $0.45 \mathrm{~mm}<\mathrm{VB} \leq$ $0.8 \mathrm{~mm}$, as shown in Fig. 4.

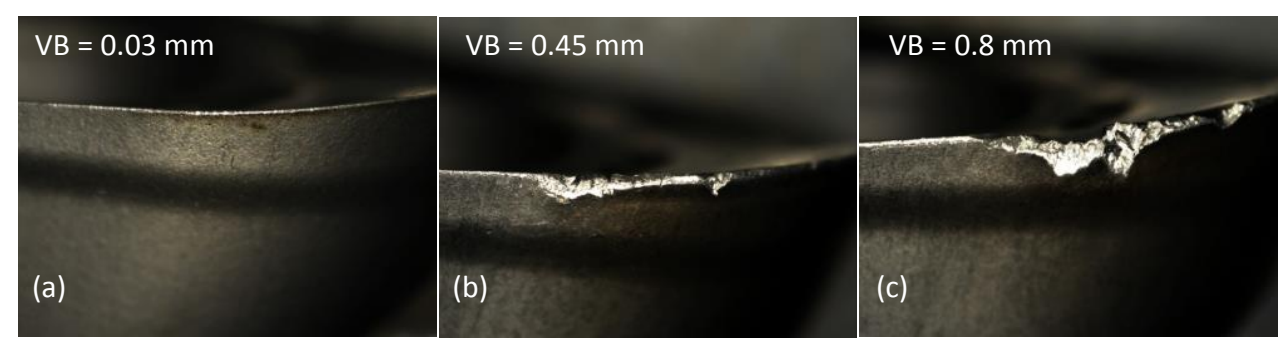

Fig. 4. Fresh (a), usable (b) worn tool (c)

\subsubsection{EXPERIMENTAL VALIDATION RESULTS AND DISCUSSION}

Figure 5 shows a time-frequency scattergram of the WSCNN features extracted using the approach developed by the authors in [13] from fresh and worn tools using three different sensors namely, a PCB accelerometer (model 356A71), the developed self-powered wireless cost-effective accelerometer, and the BECKHOFF current transducer. The shown scattergram was calculated for tool T1 during a side-milling test at $f_{z}=0.1 \mathrm{~mm} / \mathrm{tooth}, a_{p}=7 \mathrm{~mm}$, and $a_{e}=12 \mathrm{~mm}$. The effect of tool wear on the extracted features can be clearly distinguished by the high energy stored in the worn features and maintained over time. The PCB accelerometer provided a concentrated energy at the fourth passing frequency of the cutting tool, as shown in Fig. $5 \mathrm{a} 1$ and 5b1. Similar stable behaviour was obtained by the spindle current signals at the first and second passing frequencies, shown in Fig. $5 \mathrm{a} 3$ and 5b3. This would provide more stable representation of tool condition and less misclassification errors when used for tool wear state detection. However, the energy stored in the features extracted from cost-effective wireless sensor was scattered over different frequency ranges, as shown in Fig. 5a2 and 5b2. This distortion is caused by the high noise-to-signal ratio in the developed wireless sensor, as shown in the Fast Fourier Transformation FFT in Fig. 6. The electrical and seismic noises in the developed wireless sensor can be caused by the limited accuracy of the used hardware and the signal distortion during wireless transmission, which are the main drawbacks of miniature cost-effective wireless sensors. Future work should focus on overcoming such limitations as it is a building block in enabling wireless integrated TCM.

The training of the LDA classifiers for each testing set given in Table 1 was based on the features extracted from only one cut for each of the tool conditions. Each learning test was performed at the specified cutting speed $n$, minimum feedrate $f$, maximum axial depth of cut $a_{p}$ and median radial depth of cut $a_{e}$ of the testing set ranges. This significantly reduced the required learning efforts by up to $97 \%$ compared to the typical training procedures reported in literature, where up to $70 \%$ of all the acquired data are randomly selected for training $[30,34]$. 

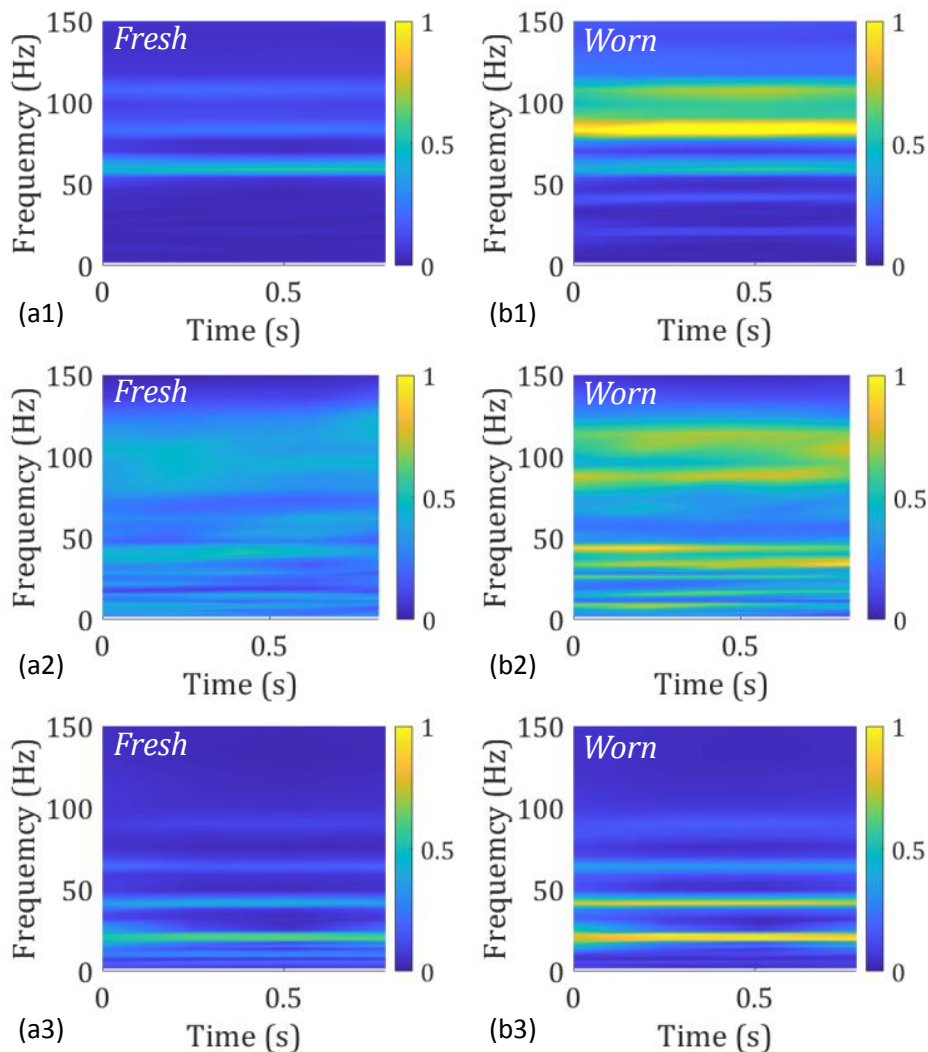

Fig. 5. Normalized scattergram of fresh(a), worn tools (b) using: (1) High-end accelerometer, (2) self-powered wireless cost-effective accelerometer, (3) current transducer
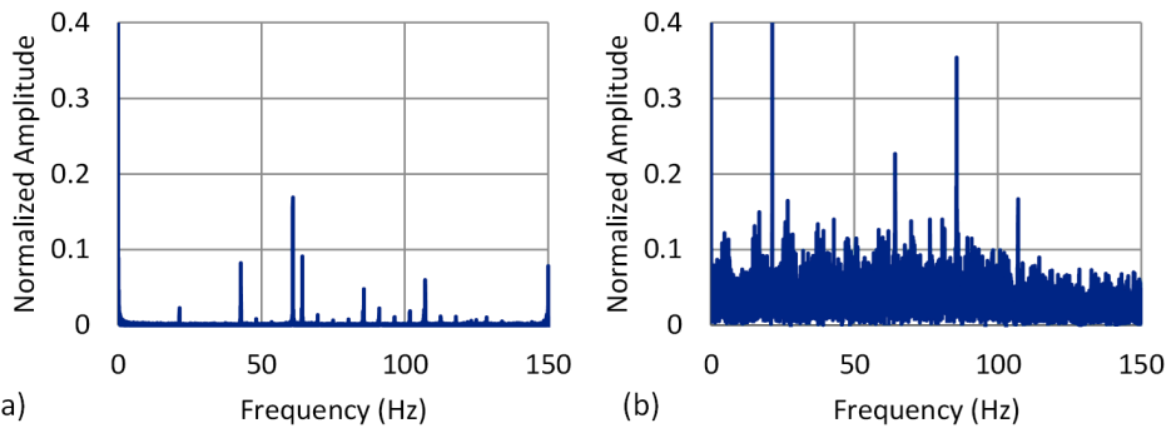

Fig. 6. FFT of vibration signals acquired using: (a) high-end wired commercial accelerometer, (b) self-powered wireless cost-effective accelerometer

In total, five classification models were developed to benchmark the WSCNN-LDA approach against different sensors and machine tool controls, as shown in Table 2. As expected, $100 \%$ accuracy was obtained using either the PCB sensor or the spindle feedback signals. This high accuracy is a result of the low variance of WSCNN features, the LDA capability to maximize separation between the tool condition clusters, and the decision making based on the triaxial vibration signals fusion in models 1 and 3, and the current, voltage and power signals fusion in model 2 and 4, which increased the approach accuracy. 
Table 2. Developed tool wear detection models and their corresponding accuracy

\begin{tabular}{lllll}
\hline \hline Model \# & Test Set \# & Sensor & Machine/Control & $\begin{array}{l}\text { Classification } \\
\text { Accuracy } \%\end{array}$ \\
\hline 1 & 1 & PCB accelerometer & Makino A88 / Fanuc 16i MA & 100 \\
2 & 1 & BECKHOFF EL3783 & Makino A88 / Fanuc 16i MA & 100 \\
3 & 2 & PCB accelerometer & DMU 100P / Siemens 840D & 100 \\
4 & 2 & LEM transducers & DMU 100P / Siemens 840D & 100 \\
5 & 2 & Wireless accelerometer & DMU 100P / Siemens 840D & 91 \\
\hline \hline
\end{tabular}

Classification model 5, which was developed based on the wireless cost-effective accelerometer, has shown $9 \%$ misclassification error. This degradation in accuracy is caused by the high noise in the acquired signals, which distorted the signal energy in the extracted features. This effect limited the ability of defining a representative mean and variance for each tool condition in the LDA developing stage.
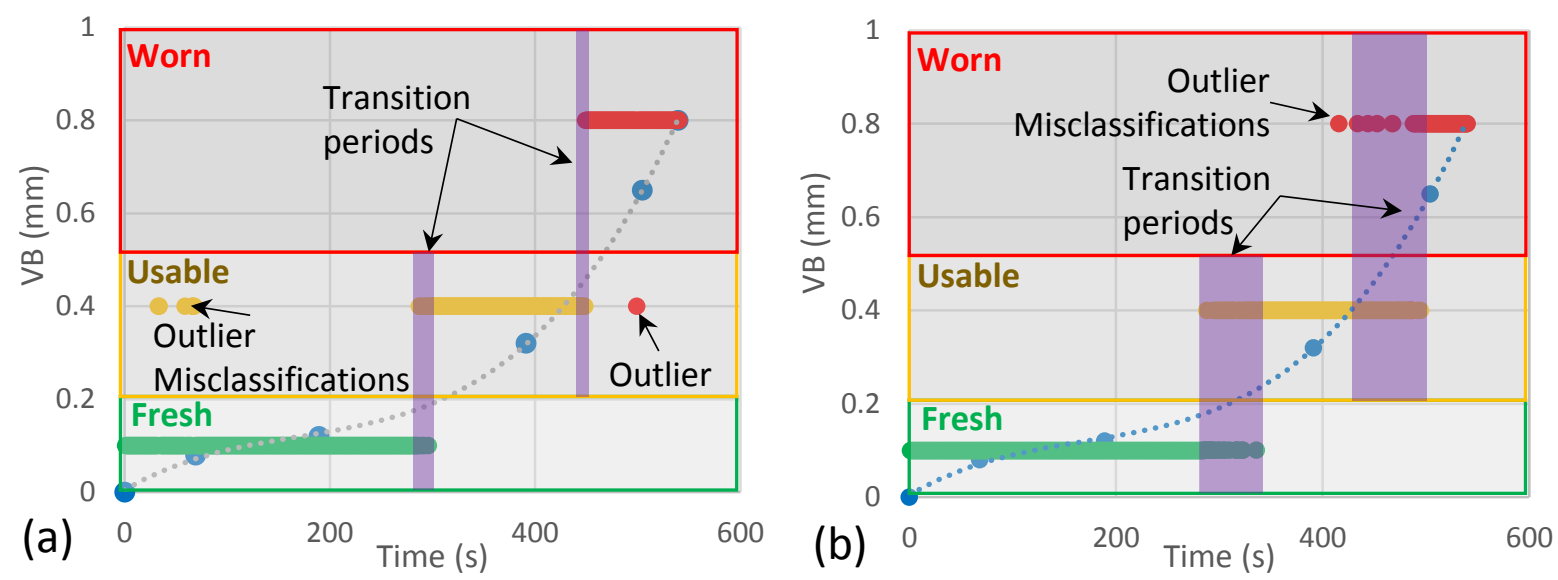

Fig. 7. Detection of tool wear evolution based on WSCNN-LDA approach using (a) spindle-mounted commercial accelerometer and (b) Spindle feedback transducers, up-milling of Ti6Al4V using T2 tool, $n=500 \mathrm{rpm}$, $f_{z}=0.12 \mathrm{~mm} /$ tooth, $a_{e}=24 \mathrm{~mm}, a_{p}=5 \mathrm{~mm}$

Figure 7 shows the detection of tool condition using the WSCNN-LDA approach over the full tool life of tool T2 when used in side milling at $n, f_{z}, a_{p}$ and $a_{e}$ of $500 \mathrm{rpm}, 0.12$ $\mathrm{mm} /$ tooth, $5 \mathrm{~mm}$, and $24 \mathrm{~mm}$, respectively. As mentioned earlier, three tool wear state classes are defined over the tool life up to $V B=0.8 \mathrm{~mm}$. In Fig. 7a, vibration signals acquired using the PCB accelerometer were used to develop the classification model, while the spindle feedback signals acquired from the BEKHOFF EL3783 were used for Fig. 7b. As seen in both graphs, the WSCNN-LDA approach has successfully defined the tool state over the tool life with acceptable misclassification outliers of $<2 \%$. However, shorter transition periods, where the detection output alters between two states near the state limits, were observed in Fig. 7a. This is due to the higher sensitivity of the vibration signals to the cutting process energy compared to the spindle feedback signals [37]. Hence, the variation of the extracted features with respect to tool condition increases, which in turn reduces the transition periods between sequential states of tool condition. 


\section{CYBER/PHYSICAL ADAPTIVE CONTROL}

In the new generation of cyber-physical systems (CPS) in machining, the process physical and computational spaces are integrated over the industrial internet of things (IIoT) network to allow monitoring, optimizing, and controlling the physical process based on information generated in both spaces and communicated therein. The physical space of the machining CPS focuses on extracting real-time reliable data that describes the relevant phenomena of the machining process, as well as the machine tool behaviour. The implementation of the physical space requires a well-designed nonintrusive sensor-based system that can effectively provide such description. In parallel, the cyber space hosts the digital twins of the machining process and the machine tool to provide additional information needed to better optimize and control the process. Such cyber space generated information is based on the real-time information extracted from the physical process; yet it has to be complementary to it through adding another level of knowledge that could not be physically measured. This involves and requires developing high fidelity physics-based predictive process models integrated with artificial intelligence and auxiliary models that facilitate reliable decision making. The effective integration of the roles of both spaces necessitates deploying a deterministic, high speed and secure network platform for sharing information in a time span that is relevant to the actual process requirements. While the CPSs aim at eliminating the errors induced by human in the loop to improve the process efficiency, the role of the human remains significant in continuously improving the intelligence of such systems. Therefore, smart Human-Machine Interfaces (HMIs) remain an important element of the cyber-physical systems.

This section demonstrates two case studies where process efficiency has been significantly improved through effective implementation of CPS.

\subsection{APPROACH FOR CYBER-PHYSICAL ADAPTIVE CONTROL OF 5-AXIS MILLING OPERATIONS}

The case study on the milling cyber-physical adaptive control system discussed herein, shown in Fig. 8, aims at increasing the process productivity while maintaining the machined part quality [42]. It fuses the physical space process information with the cyber space information from process simulation to perform closed-loop adaptive control.

In the physical space, product and process monitoring data are collected. The initial CNC machine tool settings, fixture and tool geometries, and part material are directly acquired from the CNC machine control. Different means of sensing are employed to collect processmonitoring data. These data are fed to comprehensive simulation modules of the machine tool, workpiece, cutting process and cutting tool comprised in the cyber space to evaluate the machining process productivity, the part quality, the tool geometry, and their deviation from the optimized target. System dynamics, tool life and edge geometry, tool path tracking, collision scenarios and machining time are predicted in the cyber space. The prediction outputs are fused in a process optimization module for maximizing the toolpath accuracy, collision prevention, and maintaining dynamic stability. 


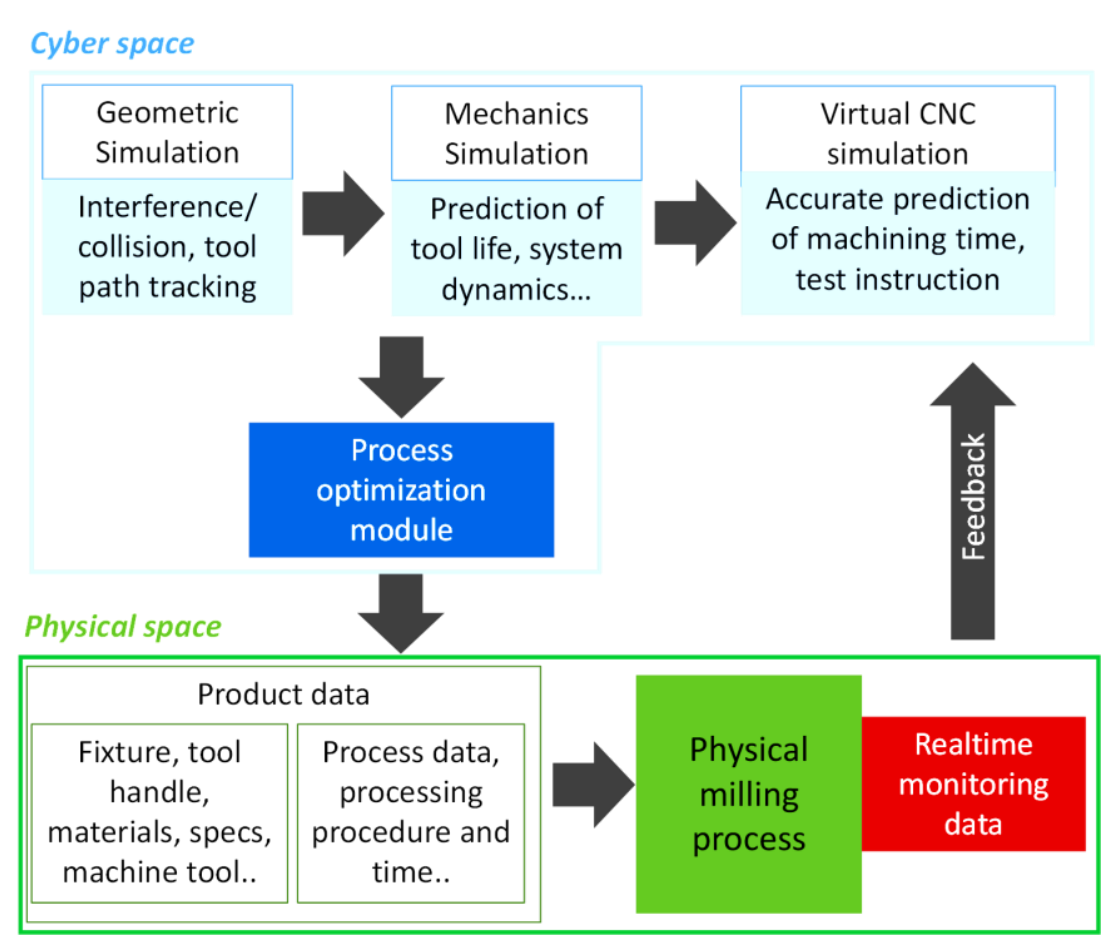

Fig. 8. Cyber-physical approach for control of 5-axis milling [42]

It adjusts the process parameters in real-time to maximize productivity and minimize the geometric and dimensional errors. In the case of tool wear evolution during the milling process, the CPS compensates in real-time for the depth of cut error through a position compensation coefficient that yields a predefined relationship with the flank wear VB value. The compensation value of the cutting depth error is estimated accordingly, and the initial error compensation is processed. After each process section, e.g., part, pass, or feature, the sensor-based system updates the actual depth of cut, which is fed to the process simulation module for part quality error evaluation with respect to the baseline reference. The optimization module generates the corresponding compensation coefficients and updates the NC program accordingly. Machining with activated error compensation is continuously reprocessed to meet the required geometric and dimensional accuracy of the machined part. The three case studies presented in [42] showed that the CPPS framework would significantly increase the CNC machining intelligence and improve the machining precision and productivity. Future studies, however, are still needed to extend the heterogeneous data scope such as process textual data and the 3D CAD models for deep learning and machining process optimization.

\subsection{APPROACH FOR CYBER-PHYSICAL ADAPTIVE CONTROL OF DRILLING OPERATIONS}

Aerostructures pre-assembly processes usually implicates drilling operations of stacked materials, which involves different tool wear mechanisms due to the tool interaction with different materials. Undetected tool wear could result in mechanical and thermal damage to the machined part due to the excessive cutting forces. Model-based tool life prediction and 
optimization systems are difficult to achieve, especially in machining of hybrid materials, due to the complexity of modelling the progressive tool wear. While available online adaptive control systems does not take into consideration the induced thermal damage and the impact of change in the feedrate on the quality attributes in machining hybrid materials. Therefore, conservative tool life criteria and cutting conditions are often used by industry to ovoid machine part scraping, which increases tooling cost and reduces productivity. To optimize the process cost, productivity, and machined part quality, model-based cyber-physical adaptive control are required. They optimize and continuously control the tool feedrate to maximize process productivity; taking into consideration, the limitations induced by tool wear level and thermal loads.

\subsubsection{CYBER-PHYSICAL ADAPTIVE CONTROL FOR DAMAGE PREVENTING AND TOOL WEAR MONITORING}

The Cyber-physical system for adaptive control of drilling of CFRPs integrates two interactive models for: (a) tool wear prediction and (b) cutting force prediction to optimize and continuously control the tool feedrate to avoid part damage by maintaining the drilling axial force below a critical level $F_{c r}$. A description of the system layout proposed by the authors is shown in Fig. 9 [43].

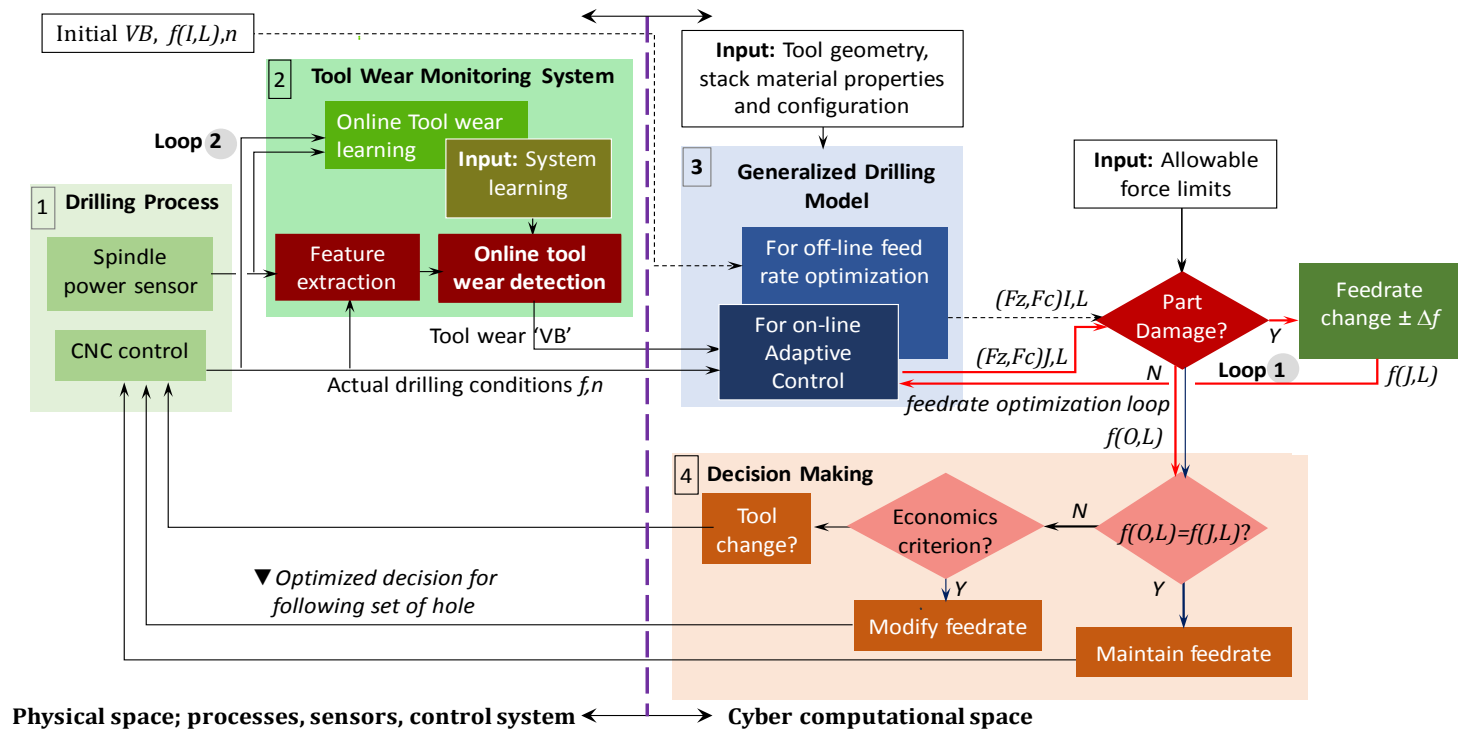

Fig. 9. Layout of cyber-physical system approach for adaptive control of drilling of hybrid stacks [43]

The process starts by inputting the initial process parameters and tool wear level to the predictive force model (box 3). Such model predicts the instantaneous cutting forces and their distributions along the anisotropic layers of the composite material throughout the drilling process, which cannot be physically measured. This enables controlling the process via comparing the predicted elemental forces $\left(F_{z}\right)$ acting on the critical material layers to the critical force limits $\left(F_{c r}\right)$ at each of such layers. This allows a more accurate and larger range 
of process enhancement than that offered when an overall force value is used. In the feedrate optimization (Loop 1), if $F_{z}<F_{c r}$, the intermediate feedrate $f_{J, L}$ is increased. The objective function of the AC system is to maximize $f_{J, L}$, constrained by the $F_{c r}$ limit, to maximize productivity and preserve part quality. The tool wear "VB" is detected (Box 2) online at the end of each hole based on the spindle power feedback signal monitored during the drilling process. The module is featured with AI-based algorithms with in-process learning capability to minimize the system learning effort needed to adapt to the varying cutting conditions. The machine controller provides the actual cutting conditions to implement their corresponding system learning. Further online learning takes place in parallel during the process using the feedback signals from the sensors and CNC controller (Loop 2). The decision-making module (Box 4) decides to either maintain or modify the feedrate to maximize the process productivity and tool life. At the instant where the negative effect of feedrate reduction on productivity exceeds the gain obtained from further using the same tool, a decision for a tool change is rendered.

\subsubsection{IMPLEMENTATION OF CYBER-PHYSICAL ADAPTIVE CONTROL OF DRILLING OF HYBRID STACKS CFRP/METAL}

The CPAC system performance developed by the authors in [43] was demonstrated for a case study of drilling a set of holes in a hybrid stacked panel, shown in Fig. 10. To maximize the process productivity, the maximum allowable feedrate for each layer, as recommended by the offline model-based optimization, was used as the initial cutting condition to drill the first hole. In this case study, the CPAC system successfully maximized the number of holes drilled by one drilling tool until its end of life, while preserving the part quality. Figure 10 shows the performance of the CPAC system, where it accurately detects the tool wear evolution after drilling each hole and accordingly shift (reduce) the feedrate to maintain the effective axial force acting on the bottom stack layer $\left(\mathrm{L}_{3}\right)$ just below the allowable critical limit to prevent part damage.

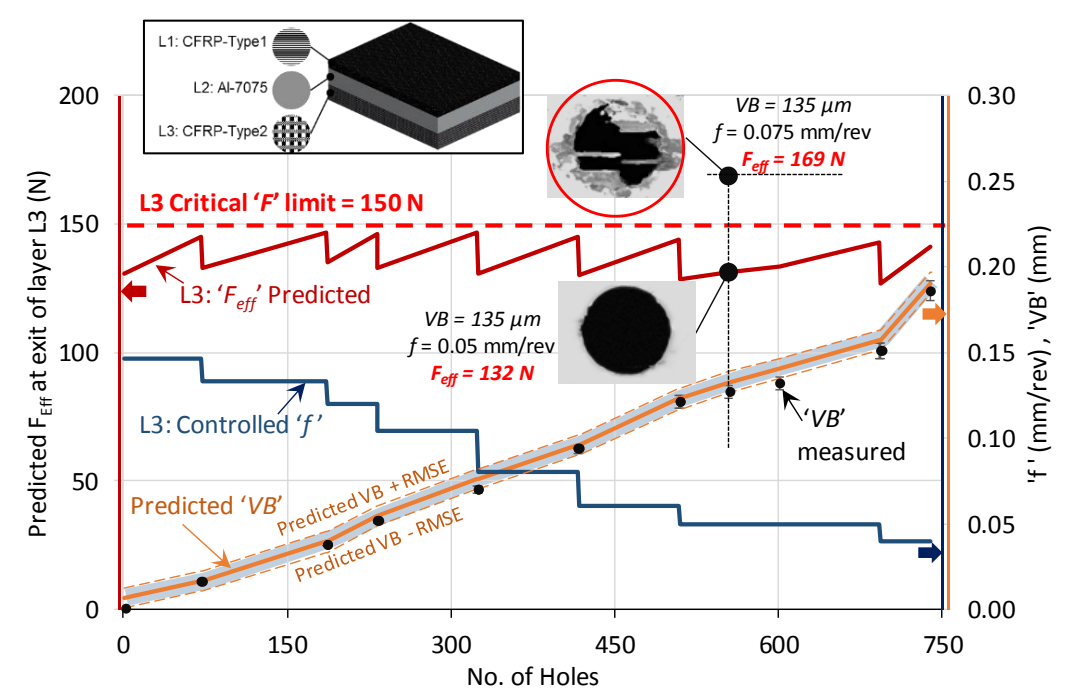

Fig.10. CPAC system performance curve with experimental validation of $V B$ and delamination predictions at exit plane of L3-CFRP [43] 
The enhancement in the process efficiency obtained by engaging the CPAC system could be quantified with respect to the conventional approach without engaging the CPAC system. In that case, seven tool changes would have been required, resulting in more than four-fold increase in cost to achieve only a marginal productivity gain of $5 \%$. The CPAC system performance was benchmarked with respect to typical criteria followed by industry that enforces pre-emptive premature tool changes to preserve part quality. This comparison showed that the activation of the CPAC system could reduce the total cycle time and process cost by up to $50 \%$ and $35 \%$, respectively.

\section{FUTURE OUTLOOK OF MACHINING DIGITALIZATION}

Today's infrastructure of industrial internet of things (IIOT) and digital systems is an evolution from a previous generation in an attempt to give insight into the manufacturing process and enable effective model-based process control through linking black box elements from the cyber and physical spaces of the manufacturing system. Digital platforms and IIoT of the factory of the future need to see a revolution where the physical and cyber spaces can closely and securely interact with the critical digital process elements throughout the different stages of the process and product life cycle stages. Quantum computing and cyber security are believed to be the game changing technologies that will breakthrough to the disruptive computation and open IIoT architectures that are relevant to digital manufacturing needs of the future.

Recent intelligent and generalized real-time TCM systems can detect and prevent sudden and progressive tool failure to safeguard the surface integrity of the machined part. They provide a generalized and practical performance and can take fast and accurate corrective actions in real-time. This improves the productivity and efficiency of machining process. However, they still require human intervention for system training, calibration, and tuning, which is timely prohibitive and increase the integration lead time. Further work is needed to facilitate the plug-and-play concept for seamless implementation in smart industrial factories.

More attention should be directed toward the development of miniature tool-embedded wireless sensors, including force, vibration, and temperature sensors. Tool-embedded sensors enable direct measurement of the primary phenomena (e.g., tool wear, tool failure) of the machining process inside the cutting zone, which provide high confidence and accurate tool condition monitoring. The wireless connection ability provides high implementation flexibility, accessibility, and connectivity. Wireless communication with machine tool control through standard protocols is also required to enable real-time control of the machining process and executing of any corrective actions needed. Additionally, it is essential to define low-variant descriptive features that are sensitive only to tool condition. This facilitates developing a new generalized in-process self-learning capability to build a dynamic database to account for the process variability and minimize/eliminate the learning process overheads. Such self-learning capability requires a novel machine learning approach to define the tool life boundaries without being trained using worn tools. The approach should maintain the logic of the monitoring process to avoid any false alarms. Moreover, system optimization is 
needed for industrial internet of things (IIoT) readiness and implementation cost minimization.

Further efforts should be directed toward the development of reconfigurable cyber physical adaptive control systems that realise dynamic fusion of offline and online optimization approaches to obtain improvement in the process productivity, while considering the thermo-mechanical and dynamic constraints imposed by the tool and workpiece interactions. This requires the development and integration of new models to predict the machining forces, temperature, and the dynamic tool behavior to identify the corresponding optimization constraints in machining processes. Such constraints can be implemented in online feedrate optimization scheme, to maximize the feedrate throughout the tool path with respect to the simulated process limits. A 2-way real-time intelligent architecture, which allows data transfer between the physical and cyber models, should take place to solve the problem of information closure between the cyber and physical spaces. This facilitates adaptive optimization of the process constraints, and hence the machining parameters, based on the current process status that are difficult to model (e.g., tool wear). A wireless plug-and-play TCM system would thrive such architecture.

Robotized machining is an essential technology in the transformation to Industry 4.0 and the efforts to establish more agile and resilient manufacturing in the Factory-of-the-future. Robotized machining has exhibited the potential to significantly increase the cost effectiveness of machining large and complex parts in low volume and highly variable batches. This is based on the fraction cost of such flexible robotic machining systems compared to that of traditional and gantry CNC machines. However, the lightweight partially supported construction of industrial robots is a main factor behind their reduced rigidity, and hence, their limited dynamic stability. Machining is a highly complex process where the dynamic stability is a key element of success. Therefore, the dynamic aspect in robotic machining involves more limitations that need to be resolved for process conditions that could have been deemed stable based on the classical stability lobes criteria. The work done on robotized machining so far over the previous years has pointed out the necessity of developing reliable cyber-physical model-based systems. This will enable controlling and reconfiguring the robotic machining platform based on the information provided collectively from the physical process feedback and the digital twin predictions to meet the targeted accuracy and productivity levels and machined part quality [44]. This necessitates developing and integrating a comprehensive accurate digital representation of the robotic machining system through its entire working envelop in terms of multi-axial models of machining forces prediction, robotic structural elasto-dynamic and dynamic characteristics of the toolworkpiece interaction. The cyber-physical optimization of the future robotic machining process will have to encompass the big data associated with all the relevant system elements including, but not limited to, the robot controller, robot arm structural dynamics, robot trajectory, machining fixture, end effector and cutting tool assemblies. This will require more robust and highly responsive robotic controllers that can keep up with the big data traversing the physical and cyber spaces in real-time to perform process adaptive control in a millisecond time range. This enables controlling the entirety of the machining system to overcome the challenges of low dynamic stability of robots while maximizing the process productivity and improving part quality. 


\section{CONCLUDING REMARKS}

This paper presented recent and future outlook of cyber-physical approaches for intelligent TCM and adaptive control systems. The advantages and limitations of wireless sensing nodes, including signal coherence and power management have been discussed and experimentally tested. A wireless vibration sensing node has been developed using miniature commercial sensors and microcontrollers. It was powered using hybrid mechanical and radio frequency energy harvesting modules. This allows non-intrusive instrumentation of machine tools and minimize the lead-time needed for system integration. Despite the practicality, simplicity and cost-effectiveness of such approach, the acquired signals showed high noiseto-signal ratio, which reduced the TCM system accuracy compared to high-end wired accelerometers. A generalized TCM approach that can define the tool condition within a wide range of cutting conditions, tool geometries and tool paths has been presented. The approach performance has shown high practicality and accuracy when tested against different types of sensors, machine controls and setups. It possesses high level of decision certainty and minimizes the learning efforts for only a couple of seconds cut per tool condition.

Two CPAC case studies have been presented, where machine, process, and product data are employed in real-time for monitoring and optimizing the cutting process in order to increase its productivity, reduce its cot, and maintain the desired product quality. Real-time reliable information from the cyber space are integrated in process mechanics and geometric simulations to predict the tool life, the product geometrical and dimensional accuracy, and the safe boundaries to boost the process productivity. Experimental validations showed such systems capability to reduce the total cycle time and process cost by up to $50 \%$ and $35 \%$, respectively, and double the tool life. These systems require using ultra-high-speed controllers to facilitate robust real-time process optimization modules to render optimization commands within the very short time span. The integration of such systems in futuristic robotized machining systems is essential to compensate the limited dynamic stability of the lightweight partially supported construction of industrial robots.

The outlook of intelligent cyber-physical monitoring and control systems in the IIOT infrastructure of I4.0 digital machining systems have been presented. The means and advantages of facilitating plug-and-play tool condition monitoring systems are described. The importance and means of reconfigurable cyber-physical adaptive control systems are discussed. The limitations of recent robotized machining systems and the possible avenues of development are listed.

\section{REFERENCES}

[1] GEISSBAUER R.SS., BERTTRAM P., CHERAGHI F., 2017, Digital Factories 2020: Shaping the Future of Manufacturing, PricewaterhouseCoopers GmbH Wirtschaftsprufungsgesllschaft (PwC).

[2] Invest in Canada - Aerospace Industries: Canada's Compatitive Advantages, 2014, Foreign Affairs, Trade and Development Canada.

[3] ZHOU Y., XUE W., 2018, Review of Tool Condition Monitoring Methods in Milling Processes, Int. J. Adv. Manuf. Technol., 1-15.

[4] ALTINTAS Y., 2014, Adaptive Control, Laperrière L, Reinhart G, editors, CIRP Encyclopedia of Production Engineering, Springer Berlin Heidelberg, 17-19. 
[5] KUTTOLAMAdOM M., 2012, Prediction of the Wear \& Evolution of Cutting Tools in a Carbide/Ti-6Al-4V Machining Tribosystem by Volumetric Tool Wear Characterization \& Modeling, TigerPrints 8, Clemson University.

[6] HASSAN M., SADEK A., ATTIA M.H., THOMSON V., 2018, Intelligent Machining: Real-Time Tool Condition Monitoring and Intelligent Adaptive Control Systems, Journal of Machine Engineering, 18/1, 5-17.

[7] LIU C., WU J-Q., LIU H-L., LI G-H., TAN G-Y., 2016, Geometry Features of Breakage Section and Variation of Cutting Force for End Mills AFter Brittle Breakage, Int. J. Adv. Manuf. Technol., 5-8, 1-14.

[8] NOURI M., FUSSELL B.K., ZINITI B.L., LINDER E., 2015, Real-Time Tool Wear Monitoring in Milling Using a Cutting Condition Independent Method, International Journal of Machine Tools and Manufacture, 89, 1-13.

[9] AMMOURI A., HAMADE R., 2014, Current Rise Criterion: A Process-Independent Method for Tool-Condition Monitoring and Prognostics, Int. J. Adv. Manuf. Technol., 72, 509-519.

[10] HASSAN M., SADEK A., ATTIA M.H, 2019, A Generalized Multisensor Real-Time Tool Condition-Monitoring Approach Using Deep Recurrent Neural Network, Smart and Sustainable Manufacturing Systems. 3/2.

[11] HASSAN M., SADEK A., DAMIR A., ATTIA M., THOMSON V., 2016, Tool Pre-Failure Monitoring in Intermittent Cutting Operations, ASME, International Mechanical Engineering Congress and Exposition, American Society of Mechanical Engineers, V002T02A49-VT02A49.

[12] HASSAN M., SADEK A., DAMIR A., ATTIA M.H., THOMSON V., 2018, A Novel Approach for Real-Time Prediction and Prevention of Tool Chipping in Intermittent Turning Machining, CIRP Annals, 67, 41-44.

[13] HASSAN M., SADEK A., ATTIA M.H., 2021, Novel Sensor-Based Tool Wear Monitoring Approach for Seamless Implementation in High Speed Milling Applications, CIRP Annals, 70/1, 87-90.

[14] MOHANRAJ T., YERCHURU .J, KRISHNAN H., NITHIN ARAVIND R.S., YAMENI R., 2021, Development of Tool Condition Monitoring System in End Milling Process Using Wavelet Features and Hoelder's Exponent with Machine Learning Algorithms, Measurement, 173, 108671.

[15] WANG G., ZHANG Y., LIU C., XIE Q., XU Y., 2019, A New Tool Wear Monitoring Method Based on Multi-Scale $P C A$, Journal of Intelligent Manufacturing, 30, 113-22.

[16] LI T., SHI T., TANG Z., LIAO G., DUAN J., HAN J., et al., 2021, Real-Time Tool Wear Monitoring Using ThinFilm Thermocouple, Journal of Materials Processing Technology, 288, 116901.

[17] TAO Z., AN Q., LIU G., CHEN M., 2019, A Novel Method for Tool Condition Monitoring Based on Long ShortTerm Memory and Hidden Markov Model Hybrid Framework in High-Speed Milling Ti-6Al-4V, Int. J. Adv. Manuf. Technol, 3165-3182.

[18] HE Z., SHI T., XUAN J., LI T., 2021, Research on Tool Wear Prediction Based on Temperature Signals and Deep Learning, Wear, 478-479.

[19] LUO M., LUO H., AXINTE D., LIU D., MEI J., LIAO Z., 2018, A Wireless Instrumented Milling Cutter System with Embedded PVDF Sensors, Mechanical Systems and Signal Processing, 110, 556-568.

[20] ZHANG C., YAO X., ZHANG J., JIN H., 2016, Tool Condition Monitoring and Remaining Useful Life Prognostic Based on a Wireless Sensor in Dry Milling Operations, Sensors (Basel), 16/6, 795.

[21] CHUNG T-K., YEH P-C., LEE H., LIN C-M., TSENG C-Y., LO W-T., et al., 2016, An Attachable Electromagnetic Energy Harvester Driven Wireless Sensing System Demonstrating Milling-Processes and Cutter-Wear/BreakageCondition Monitoring, Sensors, 16/3, 269.

[22] XIE Z., LI J., LU Y., 2018, An Integrated Wireless Vibration Sensing Tool Holder for Milling Tool Condition Monitoring, Int. J. Adv. Manuf. Technol., 95, 2885-2896.

[23] SERIN G., SENER B., OZBAYOGLU A.M., UNVER H.O., 2020, Review of Tool Condition Monitoring in Machining and Opportunities for Deep Learning, Int. J. Adv. Manuf. Technol., 109, 953-974.

[24] MOHANRAJ T., SHANKAR S., RAJASEKAR R., SAKTHIVEL N.R., PRAMANIK A., 2020, Tool Condition Monitoring Techniques in Milling Process - a Review, Journal of Materials Research and Technology, 9, 10321042.

[25] KUNTOGLU M., ASLAN A., PIMENOV D.Y., USCA U.A., SALUR E., GUPTA M.K., et al., 2020, A Review of Indirect Tool Condition Monitoring Systems and Decision-Making Methods in Turning: Critical Analysis and Trends, Sensors (Basel), 21/1, 108.

[26] WANG S-M., HO C-D., TSAI P-C., YEN C., 2014, Study of an Efficient Real-Time Monitoring and Control System for BUE and Cutter Breakage for CNC Machine Tools, Int. J. Precis. Eng. Manuf., 15, 1109-1115.

[27] HU M., MING W., AN Q., CHEN M., 2019, Tool Wear Monitoring in Milling of Titanium Alloy Ti-6Al-4 V Under MQL Conditions Based on a New Tool Wear Categorization Method, Int. J. Adv. Manuf. Technol., 104, 4112-4128.

[28] MOU W., JIANG Z., ZHU S., 2019, A Study of Tool Tipping Monitoring for Titanium Milling Based on Cutting Vibration, Int. J. Adv. Manuf. Technol., 104, 3457-3471.

[29] HUANG C.Y., LEE R.M., YANG S.K., 2016, Implement of Low Cost MEMS Accelerometers for Vibration Monitoring of Milling Process, International Conference on Applied System Innovation (ICASI), 16227947, 1-4.

[30] AGHAZADEH F., TAHAN A., THOMAS M., 2018, Tool Condition Monitoring Using Spectral Subtraction and Convolutional Neural Networks in Milling Process, Int. J. Adv. Manuf. Technol., 98, 3217-3227. 
[31] JAUREGUI J.C., RESENDIZ J.R., THENOZHI S., SZALAY T.A.J., TAKACS M., 2018, Frequency and TimeFrequency Analysis of Cutting Force and Vibration Signals for Tool Condition Monitoring, IEEE Access, 6, 64006410.

[32] JING L., ZHAO M., LI P., XU X., 2017, A Convolutional Neural Network Based Feature Learning and Fault Diagnosis Method for the Condition Monitoring of Gearbox, Measurement, 111, 1-10.

[33] YANG Y., HAO B., HAO X., LI L., CHEN N., XU T., et al., 2020, A Novel Tool (Single-Flute) Condition Monitoring Method for End Milling Process Based on Intelligent Processing of Milling Force Data by Machine Learning Algorithms, Int. J. Precis. Eng. Manuf., 21, 2159-2171.

[34] WANG P., LIU Z., GAO R.X., GUO Y., 2019, Heterogeneous Data-Driven Hybrid Machine Learning for Tool Condition Prognosis, CIRP Annals, 68/1, 455-458.

[35] CAGgianO A., RIMPAUlT X., TETI R., BALAZINSKI M., CHATELAIN J-F., NELE L., 2018, Machine Learning Approach Based on Fractal Analysis for Optimal Tool Life Exploitation in CFRP Composite Drilling for Aeronautical Assembly, CIRP Annals, 67/1, 483-486.

[36] CAI J., LUO J., WANG S., YANG S., 2018, Feature Selection in Machine Learning: A New Perspective, Neurocomputing, 300, 70-79.

[37] LAURO C.H., BRANDAO L.C., BALDO D., REIS R.A., DAVIM J.P., 2014, Monitoring and Processing Signal Applied in Machining Processes - A review, Measurement, 58, 73-86.

[38] KALVODA T., HWANG Y.R., 2010, A Cutter Tool Monitoring in Machining Process Using HilbertHuang Transform, International Journal of Machine Tools and Manufacture, 50/5, 495-501.

[39] WESTERMARK P., 2017, Wavelets, Scattering Transforms and Convolutional Neural Networks: Tools for Image Processing, Uppsala University.

[40] ANDEN J., MALLAT S., 2014, Deep Scattering Spectrum, IEEE Transactions on Signal Processing, 62, 41144128.

[41] CUKA B., KIM D-W., 2017, Fuzzy Logic Based Tool Condition Monitoring For End-Milling, Robotics and Computer-Integrated Manufacturing, 47, 22-36.

[42] ZHU K., ZHANG Y., 2018, A Cyber-Physical Production System Framework of Smart CNC Machining Monitoring System, IEEE/ASME Transactions on Mechatronics, 23, 2579-2586.

[43] SADEK A., HASSAN M., ATTIA M.H., 2020, A New Cyber-Physical Adaptive Control System for Drilling of Hybrid Stacks, CIRP Annals, 69/1, 105-108.

[44] JI W., WANG L., 2019, Industrial Robotic Machining: a Review, Int. J. Adv. Manuf. Technol., 103, $1239-1255$. 